\title{
Research of Auxiliary Recognition System of Image for the Blind Based on Tactile Perception
}

\author{
Zhang Qinghui $^{1, *}$, Zhu Zhiyuan $^{1}$ and Zhang Donglin ${ }^{2}$ \\ ${ }^{1}$ Henan University of Technology, Zhengzhou, Henan, 450001, China \\ ${ }^{2}$ Zhengzhou Municipal Bureau of Education, Zhengzhou, Henan, 450001, China
}

\begin{abstract}
In order to help the visually impaired to perceive images with tactile mode, a tactile perception system is designed, which used image acquisition module to analog shape, color and luminance information of the object seen by human eyes in the surrounding environment, and analyzed and processed the information through the data processing module. Through the different control signals, the data processing module controlled tactile producing device and current producing module to produce the current of different region, frequency and intensity to stimulate the body skin of user in certain parts, thus transferring the included shape, color and luminance information of image information to user. The system can help the visually impaired to perceive the image information.
\end{abstract}

Keywords: Auxiliary recognition, blind person, human abdomen, image information, tactile perception.

\section{INTRODUCTION}

For the visually impaired due to the loss of vision, accessing to information mainly relies on the touch and hearing for catching image information, which is otherwise hard to perceive effectively. How to let the blind access to a certain degree of image information is the problem that the researchers should pay attention to. The sense of touch is the most important way for visually impaired to gain access to environmental information. Through tactile stimuli that can be formed of rich "Tactile Language" according to certain rules of combination, realized the tactile expression and perception. Medical research shows that the visual cortex of the blind, still retains the visual information analysis function organization, and the information interaction is obtained through the parietal cortex of the brain. Although the blind irritants in the treatment of the external space information are not done directly by the visual cortex, yet it is completed by hearing and touch. But looking at clinical trials, the blind of the visual cortex can be activated and reused in order to help the blind perceive the world better, which provides the reason for the basic theoretical basis of this paper [1].

In order to realize the actual touch of image information, this paper puts forward a kind of system that can automatically collect image information and convert to electrical stimulation of tactile perception. Based on ARM system design, the image information acquisition, RGB information extraction, stimulates the production of electric current and tactile information. The system works through the image acquisition module to collect the object shape, color and brightness information, which are stored with the RGB pixel format after recodification, and every pixel color and luminance

*Address correspondence to this author at the Henan University of Technology, Zhengzhou, Henan, 450001, China; Tel: +8615638425000; E-mail: 196999757@qq.com information is converted into a different intensity, frequency and area of the current stimulus signal to make abdominal skin irritation to human body, letting the wearer perceive image information by tactile stimuli. Therefore, matrix intelligent blind graphics perception process of tactile sensing device is more close to the actual process of human visual perception; it shows the substantial advance in the research of image information to understand the meaning of existing visual perception, also that the connection between the touch nerve and the optic nerve is a conversion process giving a new research subject [2-4].

\section{IMPLEMENTATION METHOD}

\subsection{The Basic Principle}

The blind image aided identification system is based on tactile perception; that is based on the principle that the human eye visual imaging, uses CCD video image acquisition, image edge detection and visual pixel heavy coding and color - frequency conversion algorithm, combined with the principle of abdominal skin conditioning through image information encoding, using the array type current, stimulates the abdominal skin for the blind. After the blind man gets professional training, the blind man will be in a characteristic way perceive the environment of the physical shape, color, brightness, and near or far, and auxiliary. This perception of the world will help them improve the quality of their lives. This research contents include: visual images transformation into auxiliary tactile recognition methods, the research on target recognition algorithm under complicated background, designing a kind of RGB pixel colors to touch frequency conversion algorithm, studying the human body under the current tactile stimulus neurophysiological parameters, and visual and tactile perception interaction with the information [5]. 


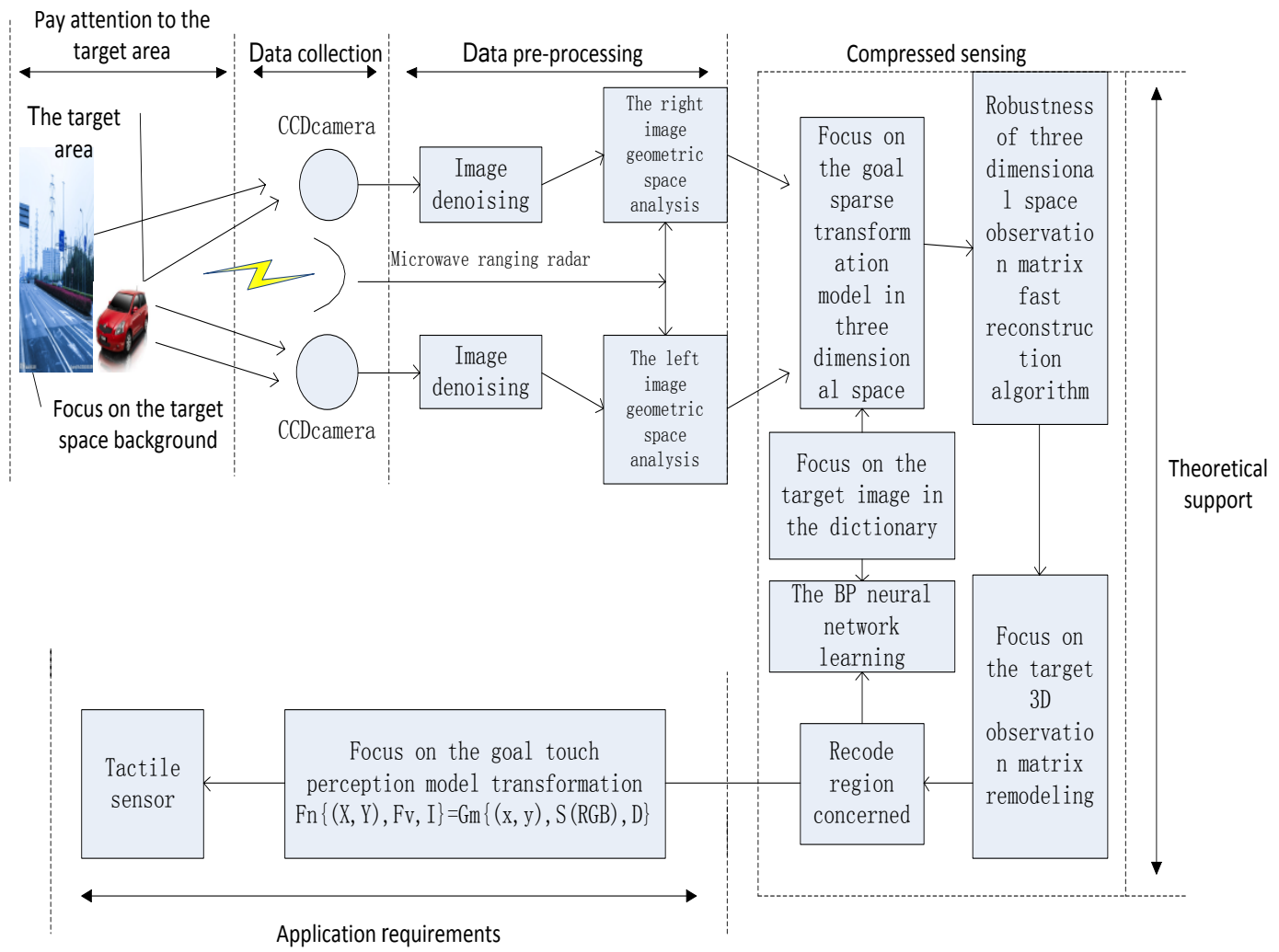

Fig. (1). The whole solution of the system.

\subsection{The Method of Visual Images into Touch Aided Identification}

In the study of the system, our aim is to solve the blind identification of visual objects through touch imaging information, therefore we can imitate the human eye visual cognitive process. According to the physiological and psychological characteristics of the blind with respect to visual perception mechanism one should focus on how to mimic this converted image information to the blind identifiable tactile information. In this process, we need to carry out research on three important points: firstly, in terms of visual way to collect information, how to imitate the human eye optical imaging principle, such as automatic focusing and parallax angle according to the human eyes fuzzy matching algorithm for $3 \mathrm{D}$ vector graphics model; secondly, since the human body skin resolution and visual resolution are inconsistent, so how to build the two pixel information of the best mapping model, namely, the heavy coding; and thirdly, that the blind tactile identification process is a dynamic tracing process, so how to ensure the stability of target imaging and real-time acquisition system that it can identify the error feedback to see the road to adjust the focus on the goal clarity.

\subsection{The Research Focus on Target Identification Algo- rithm Under Complex Background}

Focusing on the target recognition is a unique function of human eyes, which includes: imitating human visual perception process, paying attention to focus on the target selection algorithm as the core, ensuring the dynamic adjustment focus on the target imaging area, ensuring real-time acquisition and processing, paying close attention to the target area pixel for RGB colors and tactile frequency mapping relation mod$\mathrm{el}$, and realizing the goal that visualizes the visual sense of touch information reprogram $[6,7]$. The whole solution system is shown Fig. (1).

In order to strengthen the focus on the target outline and details, use of the following method to deal with focus areas is suggested:

\subsubsection{Selective Target Image Edge Detection and Search Target Matching Algorithm}

Selective image edge detection mainly refers to the complex image background, according to the interest in the target image like gray scale, texture and color change of discontinuity. We use a variety of algorithms to extract the information of target area of the image characteristic value, where it can keep the selected target's shape, color and brightness information.

\subsubsection{Visual Images-Tactile Perception Coding Algo- rithm}

The purpose for visual images conversion into touch perception coding based on visualization technique, is to convert the image pixels for obtaining the blind touch perceived frequency of contact. The corresponding relation between contact frequency and visual pixel is:

$\mathrm{Fn}\{\mathrm{X}, \mathrm{Y}, \mathrm{Fv}, \mathrm{I}\}=\mathrm{Gm}\{\mathrm{x}, \mathrm{y}, \mathrm{S}(\mathrm{R}, \mathrm{G}, \mathrm{B}), \mathrm{D}\}$ 
where Fn is blind skin contact matrix where parameters of $\mathrm{X}$, $\mathrm{Y}$ that are location coordinates, Fv the color at a certain frequency, current I represents point distance (brightness); Gm is a visual image matrix where the parameters of $x, y$ are the pixel coordinates; $\mathrm{S}(\mathrm{R}, \mathrm{G}$, and $\mathrm{B})$ is trichromatic pixel point; $\mathrm{D}$ is the visible distance.

Because of the physiological differences between visual and tactile, visual information of pixels and tactile perception information point have a nonlinear corresponding relation. So, you need to strengthen encoding and weaken coding of view area according to the focus area and not the focus area, to ensure that the focus on the target area of tactile perception pixel density is higher. About nonlinear scaling factor of the two, one needs to decide by the tactile dot density. In view of this, one needs to choose to focus on the goal of image edge detection and contour extraction of characteristic value. Through experiment, we proposed the ascension based wavelet image edge detection algorithm.

\subsubsection{D Image Visual Observation Information Compres- sion Reconstruction Algorithm}

To build an ideal space, observation matrix is as follows:

$\mathcal{F}(\mathrm{x}, \mathrm{y}, \mathrm{d})=\sum \alpha_{\mathrm{i}} \psi_{\mathrm{i}}=\psi \alpha$

where $\mathcal{F}$ is sparse compressible ideal observation space coordinate information, $\psi=\left\{\psi_{1} \psi_{2} \cdots \cdots \psi_{\mathrm{N}}\right\}$ is a sparse change matrix, $\alpha_{\mathrm{i}}$ is sparse coefficient. Because in CS code measurement model, sparse signal $\mathcal{F}$ is not directly measured, but through the signal $\mathcal{F}$ projection on a set of measurement matrix, then measurement matrix is obtained as follows:

$y=\psi \mathcal{F}(x, y, d)$

So that:

$y=\phi \mathcal{F}(x, y, d)=\phi \psi \alpha$

With the noise, spatial structure observation model building is as follows

$y=\mathcal{F}(\mathrm{x}, \mathrm{y}, \mathrm{d})+\varepsilon(\mathrm{x}, \mathrm{y}, \mathrm{d})=\psi \alpha+\varepsilon(\mathrm{x}, \mathrm{y}, \mathrm{d})$

So, the problem is transformed into the Lagrange multiplier method which is adopted to solve the following optimization problem of the model.

$\mathcal{F} \mathrm{c}(\mathrm{x}, \mathrm{y}, \mathrm{d})=\min \|(\mathrm{y}-\psi \alpha-\mathcal{E}(\mathrm{x}, \mathrm{y}, \mathrm{d}))\|_{2}^{2}+\lambda\|(\alpha)\|_{1}$

$\mathcal{F}_{\mathrm{C}}$ is refactoring coordinate, $\lambda$ can adjust the balance between the permissible error and sparse [8].

\subsection{Three Primaries Frequency Conversion}

Central processing unit will convert the color and brightness information into different intensity and frequency current signals. Experimental results show that eyes within the visible spectrum have different sensitivity response for different wavelengths of radiation (as shown in Fig. (2)). Through the spectrum curve, it is known that the human eyes are sensitive to yellow and green wavelengths $(550 \mathrm{~nm})$. In order to touch this phenomenon with more accurate frequency sensitivity of nonlinear analog, the conversion formula is:

$F_{V}=F_{b} \times B+F_{g} \times G+F_{r} \times R$
Among them, $F_{b}, F_{g}$ and $F_{r}$ are frequency conversion coefficients of $\mathrm{R}, \mathrm{G}, \mathrm{B}$ respectively. These coefficients have non-linear relationship. But, they need to be adopted based on the least squares method and spectral luminous efficiency curve for approximate determination of the conversion coefficient.

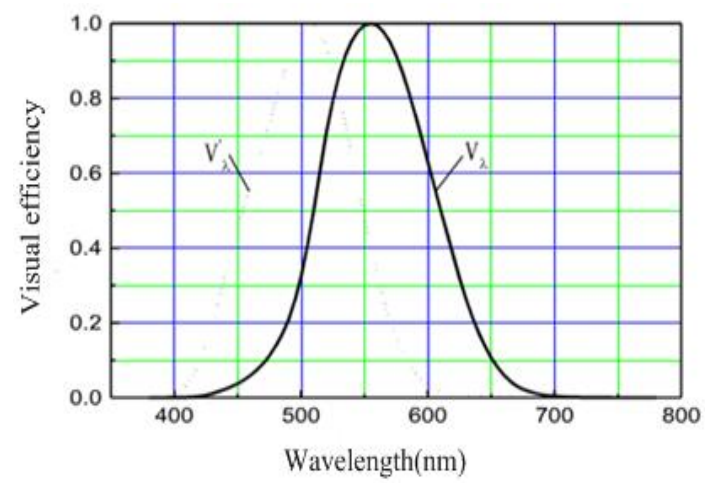

Fig. (2). The relationship between wavelength and visual efficiency.

\subsection{Abdominal Tactile Sensitivity Tests to Current Stim- ulation of the Human Body}

The key of this project is to determine human abdomen tactile sensitivity under the effect of different currents and flings of threshold current intensity and frequency change.

\subsubsection{Signal Generator}

The human skin tissue extends as a result of load application. The system design of the current intensity and frequency signal source may undergo continuous variation within a certain scope, and its skin impedance parameter value may be uncertain due to differences in human skin tissue. In order to produce a constant PWM modulation current value, we used the microcomputer constant current controller. As shown in Fig. (3), it is a PWM constant current control block diagram. Through the circuit current intensity and PWM pulse width modulation control system, constant current and the stimulation of different frequency pulses could be obtained. First, according to the setting of current intensity, frequency of color, V/I and PWM constant current output a, the protection circuit sends out the signals. Then, the CPU collects the feedback signal and adjusts the output signal to ensure the skin impedance change on human skin.

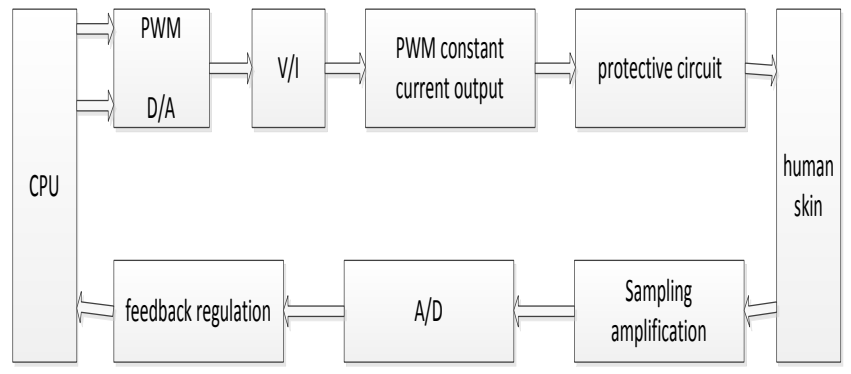

Fig. (3). The block diagram of PWM modulating constant current. 


\subsubsection{Signal Extraction}

Frequency resolution threshold is detected with reference to the commonly used threshold current detection algorithm of nervous system, namely UDTR rules method. To gain the effective basic data, we selected three individuals to participate in the test. Fig. (4) shows the blind touch points frequency resolution test data, designed to test relation between frequency and the body color sense at different frequencies. From the test results analysis, test data and the basic theoretical calculation, it has been observed that the difference in frequency of the crowd tactile band obtained through overall migration test, will not affect the use effect of the system. Test conditions are set at a current strength of $2.5 \mathrm{~mA}$, gradually adjusting the frequency (from 0 to $1500 \mathrm{~Hz}$ continuous stimulation). From the test data analysis, it has been suggested that the lower the frequency is, the higher the sensitivity of human body to frequency change, thus they are conversely proportional. When the frequency is greater than $1500 \mathrm{~Hz}$, human abdomen cannot distinguish less than $50 \mathrm{~Hz}$ frequency change quantity. This also shows that the sensor's touch frequency band selection is in the range of $0-1100 \mathrm{~Hz}$ [9].

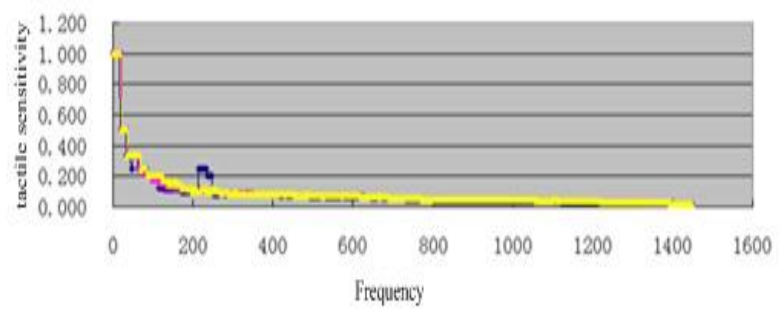

Fig. (4). The experimental data of human abdominal skin touch frequency change threshold.

\section{CONCLUSION}

In this paper, we designed a kind of auxiliary tactile image perception system for the blind, using image acquisition module to simulate human eyes to see objects in the form of shape, color and brightness, and processing of the surrounding environment through the analysis of the data processing module. Then, the data processing module generates touch device and current control module by different control signal. It is suggested that specific parts of the stimulated body skin of the users should be applied different frequency and intensity of current, so the information containing image is transmitted to the user by the shape, color and brightness information. The system can help the visually impaired by transmitting sensory image information in a visual way. In the future, we will further improve the tactile coding method, to expand the practicability of the system [10].

\section{CONFLICT OF INTEREST}

The authors confirm that this article content has no conflict of interest.

\section{ACKNOWLEDGEMENTS}

This work is supported by the National Natural Science Foundation, China (No.u1404617). The National Science and Technology in rural areas of "the Twelfth Five-Year Plan", China (2013BAD17B04-1).

\section{REFERENCES}

[1] A.M. Simon, L.J. Hargrove, B.A. Lock, and T.A. Kuiken, "Target achievement control test: Evaluating real-time myoelectric patternrecognition control of multifunctional upper-limb prostheses," Journal of Rehabilitation Research and Development, vol. 48, no. 6, pp. 619-628, 2011.

[2] M. Loog, "Semi-supervised linear discriminant analysis through moment-constraint parameter estimation," Pattern Recognition Letters, vol. 37, no. 1, pp. 24-31, 2014

[3] G. Cerutti, L. Tougne, J. Mille, A. Vacavant, and D. Coquin, "Understanding leaves in natural images - A model-based approach for tree species identification," Computer Vision and Image Understanding, vol. 117, no. 10, pp. 1482-1501, 2013.

[4] D.P. Gierga, J. Brewer, G.C. Sharp, M. Betke, C.G. Willett, and G.T.Y. Chen, "The correlation between internal and external markers for abdominal tumors: Implications for respiratory gating," International Journal of Radiation Oncology Biology Physics, vol. 61, no. 5, pp. 1551-1558, 2005.

[5] B. Gao, and W.L. Woo, "Wearable audio monitoring: Contentbased processing methodology and implementation," IEEE Transactions on Human-Machine Systems, vol. 44, no. 2, pp. 222-233, 2014.

[6] P. Liu, and L. Li, "Hard thresholding gradient pursuit of compressed sensing," Journal of Computational Information Systems, vol. 10, no. 13, pp. 5713-5721, 2013.

[7] K. Arai, and K. Okajima, "Surround effects affecting roughness in tactile perception," Journal of the Institute of Image Information and Television Engineers, vol. 63, no. 12, pp. 1800-1806, 2009.

[8] M. Ding, L. Wei, and B. Wang, "Research on fusion method for infrared and visible images via compressive sensing," Infrared Physics and Technology, vol. 57, pp. 56-67, 2013.

[9] X.D. Cai, and Z.G. Liu, "Signal processing circuit design and application for infrared optical system based on FPGA," Applied Mechanics and Materials, vol. 347-350, pp. 1328-1332, 2013.

[10] H. Hu, and R. Ding, "Least squares based iterative identification algorithms for input nonlinear controlled autoregressive systems based on the auxiliary model," Nonlinear Dynamics, vol. 79, no. 1, pp. 777-784, 2014.

(C) Qinghui et al.; Licensee Bentham Open.

This is an open access article licensed under the terms of the (https://creativecommons.org/licenses/by/4.0/legalcode), which permits unrestricted, non-commercial use, distribution and reproduction in any medium, provided the work is properly cited. 